\title{
Wireless Networking for Virtual Immersive COMmunications: The VICOM Project
}

\author{
F. Davide ${ }^{1}$, A. Detti ${ }^{2}$, E. Gregori ${ }^{3}$, and F. Vatalaro ${ }^{4}$ \\ ${ }^{1}$ Telecom Italia Learning Services \\ Rome, Italy \\ Fabrizio.Davidedtelecomitalia.it \\ ${ }^{2}$ CNIT - University of Rome "Tor Vergata", \\ Italy \\ andrea.detti@uniroma2.it \\ ${ }^{3}$ IIT Institute of the Italian National Council, \\ Pisa, Italy \\ enrico.gregori@it.cnr.it \\ ${ }^{4}$ University of "Rome Tor Vergata", \\ Electronic Dept., Italy \\ vatalaro@ing.uniroma2.it
}

\begin{abstract}
The paper describes the Virtual Immersive Communication (VICOM) project, and reports the state of art with respect to the wireless networking issues. The project vertically exploits the enabling technologies for Immersive communications enriched with virtual reality components. The research topics are common driven toward the final project goal, consisting in proof of concept demonstrators: Mobility in Immersive Environments (MIE) and Virtual Immersive Learning (VIL).
\end{abstract}

\section{Introduction}

The increasing relevant role of multimedia mobile communications, joined with the emerging technologies on audio, video and sensing interfaces, makes challenge their integration, in order to realize novel form of human interactions at distance. Thus it is possible to create a sense of being in the remote place and to make natural, simple and continuous the interactions with the ambient. The first characteristic is often referred as telepresence, while the other is referred immersive service.

With this regard, some building blocks necessary to realize these services, consist in: i) multi-modal service interfaces aware of the user context; ii) wireless and sensor networks; iii) middleware platforms for mobile applications.

The multi modal interfaces allow the user to interact with the system in natural ways (e.g., voice, gesture, etc.); moreover, they output such realistic multimedia contents that create the sense of telepresence. The awareness of the user context saves in the retrieval of information from the user, therefore simplifies the service accessibility [2]; the previous ones joined with the continuous opportunity in dialing with the system, give to the user the sensation of the continuous presence of the service around him; i.e. "immersivity". 


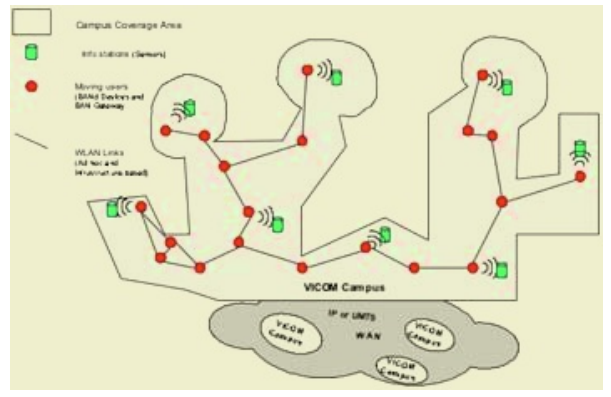

Fig. 1. VICOM network reference scenario

In these research areas, some italian partners, (universities, governemnet research centers and private companies), started the Virtual Immersive COMmunication Project $(\mathrm{VICOM})^{1}$ [1]. The project goal is to study and development solutions for ambient intelligence systems and to prepare two demonstrative scenarios. These scenarios are named Mobility in Immersive Environment (MIE) and Virtual Immersive Learning (VIL), and are aimed at stressing complementary services aspects; respectively, "immersivity" and "telepresence".

To provide the immersivity functionalities, MIE scenario mainly exploits advanced mobility issues. It is assumed that the user wears a set of devices forming a body area network (BAN). One of these performs the gateway functions towards the surrounding Personal Area Network (PAN) and with Wireless Local Area Network (WLAN). In order to provide a seamless connectivity in different PAN/WLAN environment, the BAN gateway is able to reconfigure its low layers (up to the data link), via software radio mechanisms. These services will provided within an area of limited coverage, e.g., a campus. Communications between BANs is obtained both with access base stations (infrastructure-based WLAN) and with ad hoc solutions, see Fig. 1.

The resulting service coverage defines the VICOM Campus. Within the campus, a set of sensors provides the user contextual. Finally, the campuses are together interconnected by means of IP Wide Area Network (WAN).

VIL scenario foresees that the users join a tele-training session, enriched by virtual reality contents, and exploit the telepresence concept.

In the next sessions, we report the network topics dealt within the VICOM project in order to support the proposed demonstrators.

\section{VICOM Networking: State of Art}

In both scenarios, we have to integrate the several wireless networking technologies (for BAN, WLAN end Sensors) to provide connectivity among user within the room

1 The VICOM Project is funded by the Italian Ministry of Instruction University and Research (MIUR). It is three years long, and it is started in the end of 2002. The partnership is formed by: the CNIT universities consortium; the Pisa and Bologna units of the National Research Center (CNR); the "Politecnico di Milano"; the ISCTI national institute and Telecom Italia Learning Services. 


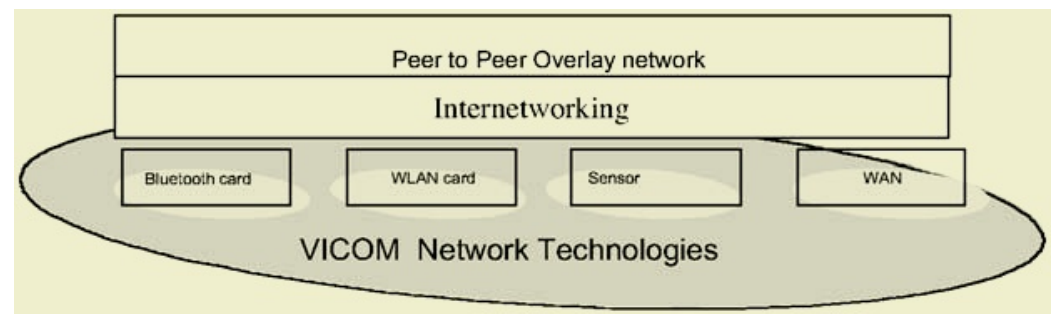

Fig. 2. Sketch of VICOM protocol architecture of the fixed network devices (WAN), of the gateway sensor devices, of the BAN gateway (Bluetooth and WLAN IEEE 802.11)

or the campus and connectivity with remote user via Internet; anyway, the different scenario requirements split the research directions. Due to the wideness of service coverage, in the MIE scenario the focus is placed on solution for mobility as Quality of Service is not critical. On the other hand in the VIL scenario we have the opposite situation: Quality of Service management is a strong requirement (we have high bandwidth requirement from the application) while mobility is not critical due to the limited coverage of the services (e.g., an equipped room).

The target of the VICOM project is to set up two proof-of-concept demonstrators; hence, the research topics are strongly tied with the off-the-shelf network technologies. The networking research is focused on the Bluetooth technology for the BAN/PAN, on 802.11 for the PAN/WLAN and on a not yet defined technology for sensor networks. As consequence, from a technology point of view, VICOM faces internetworking problems with four network types (sensor, Bluetooth, IEEE 802.11 and WAN). The networking and QoS strategies within the specific area will be customized according to the technology features and employment (Fig. 2). A coarse customization, instead, will be performed at the transport layer, where the issues coming out for the TCP unsuitability with the wireless link will be probably solved in a technology independent fashion, by developing a "wireless TCP" suitable both for Bluetooth and IEEE 802.11 and whenever needed adopting indirect-TCP solutions. Nevertheless, for those devices that do not require to widely be accessible (such as the membership of a BAN with the exclusion of the BAN gateway), it is not sure that those IP compliant transport and routing techniques will be necessary, and so other possible technology-specific protocols "non-ip compliant" have to be taken into account.

At least for the wireless gateway, we envisage a possible protocol differentiation up to the transport level that requires some bridging layer functions to limit the negative impact of wireless technologies on the need-to-end performances. For this reason we will introduce some proxy nodes in our networks. In addition we need some middleware services. The most important is the many to many multimedia communications, which is a basic requirement of cooperative work applications, that is obtained constructing a peer-to-peer overlay network.

In the following sections, we report the VICOM research lines more tied with the wireless networking issues and describe the topics of the current research. 


\subsection{IEEE 802.11}

In this section, we report the technical novelties that VICOM project is currently addressing in order to enable the IEEE 802.11 wireless infrastructure and ad hoc networks to fulfill the MIE and VIL application requirements. These topics are dealt both by means of analytic and simulation tools; hence, for the more promising solutions, some "on-the-field" performance measurements are on going [4].

\subsubsection{Ad hoc Routing Protocols}

For the applications envisioned in the VICOM scenarios, and more specifically for MIE, it is important to have mechanisms, which make it possible to establish data communications even beyond the coverage of the fixed infrastructure. It is therefore appealing to use the ad hoc networking technology, where nodes flexibly collaborate in delivering the information to the final destination. Due to its natural broadcast characteristics, the IEEE 802.11 is a promising and available technology to realize an ad hoc network. With this regard, a VICOM research line is currently focused on routing in ad hoc networks, with special effort on energy efficiency of the considered schemes [5] and in contrast with the intuitive goal of choosing the shortest paths, actually accomplished by the well know AODV and DRS ad hoc routing protocols.

\subsubsection{Quality of Services Mechanisms}

As previously stated, most of the VICOM services require the delivery of multimedia data, like streaming video and audio. This feature needs the support of time-bounded services by the infrastructure or by ad hoc network. Nevertheless, the legacy IEEE 802.11 standard was designed to support only best-effort services. Therefore, a careful analysis is coming on to verify the applicability of two mechanisms, both discussed in the latest version of the 802.11e draft standard: the Enhanced Distributed Coordination Function (EDCF) and the Hybrid Coordination Function (HCF).

\subsection{Bluetooth}

Although IEEE 802.11 technology furnish higher bandwidth respect to the Bluetooth (BT) one and so, it appears as more suitable for multimedia applications, we have to take in mind that IEEE 802.11 drains a significant amount of energy and processing resources often not available on small devices as PDA [6]. On the contrary, Bluetooth owns an high electronic integrability, a low power drain and a low device processing requirement being a lot of the computation directly implemented on the radio chip; that is more or less witnessed by the market penetration of Bluetooth in small and low performance devices such as PDA, mobile phones and earphones. For these reason the Bluetooth technology is the more promising candidate for the BAN, but it has to be considered also in the realization of "simple" device PAN, with or without infrastructure.

In the following, we report the main Bluetooth research topics currently dealt in VICOM project. 


\subsubsection{Scatternet Formation Algorithms}

While the ad hoc communication are quite natural in IEEE 802.11, this doesn't occurs in Bluetooth; hence, suitable scattered formation protocols have to be developed [7]. Both scatternet formation protocols that require the radio vicinity of all nodes, for BAN and infrastructured PAN, and protocols working in the more general multi-hop scenario, for ad hoc PAN, are taken into account. Moreover, major focus is placed on distributed techniques, in the sense that the protocols are executed at each node with limited knowledge of the surrounding topology.

\subsubsection{Handover and Fast Connection Restablishment}

Most of Bluetooh communications are based on L2CAP connections, unlike with the connectionless nature of IEEE 802.11 MAC layer. This difference, joined with the slowness of the inquiry procedure [8], leads the need for the Bluetooth research line to investigate on fast and reliable handover and in fast connection reestablishment protocols. The former may be accomplished for those devices that sojourn within the radio coverage during the movement and are able to simultaneously belong to two or more piconet (i.e. are able to perform the scatternet functionalities); hence, they can hand over from one piconet to the other without breaking the connectivity. In the other cases, e.g. in presence of shadowing or of device not quite able to manage advanced scatternet functionality, fast connection restablishment mechanisms may be candidate to avoid the high layer protocol timeout (e.g. TCP, HTTP and so on).

\subsubsection{Ad hoc Routing Protocols}

As done in the IEEE 802.11 research line, Ad hoc Routing Protocols that take into account of Bluetooth technology characteristics are dealt in VICOM project. Currently, the major focus is based on the optimization of the following protocols: the Broadcast Incremental Power (BIP), the Broadcast least unicastcost (BLU) and the Broadcast Link-based MST (BLiMST) algorithm.

\subsection{Sensor Networks}

During the description of VICOM scenario, we show the opportunity in using sensor networks to retrieves the relevant contextual information. Sensor networks are inherently resource poor, i.e., they are fundamentally constrained in terms of CPU power, energy, memory. This calls for new design in routing techniques since, e.g., complicated routing protocols cannot be loaded onto sensor nodes due to the lack of memory to store the code and possibly due to the excessive amount of computations they may involve. Also, storing locally large routing tables is impractical and may not be feasible due to memory constraints [9]. As consequence, the current VICOM research on sensor networks are focused on energy efficient routing protocols that differs form the Bluetooth and IEEE 802.11 ones in terms of lower complexity to the detriment of routing performance, which, however may be acceptable due to the small network load. 


\subsection{Upper Layer Protocol}

Above the routing layer, the transport and the overlay protocols form what we call upper layer protocols. These protocols may support the applications layer, both directly or through the middleware level.

\subsubsection{Wireless TCP}

Wireless links are characterized by a bit error rate, which is much higher than in wired links, and segment losses may occur due to link errors rather than congestion [10]. The transmission rate decreases caused by segment losses due to link errors are unnecessary and only result in a decrease of the throughput performance. This is the major cause of the scarce TCP efficiency in all wireless communication environments. VICOM research are facing this issues with two approaches: i) adaptation of TCP congestion control algorithm to the wireless environment; ii) decrease in the wireless link bit error rate through the use of appropriate error correcting schemes and power management.

\subsubsection{Peer to Peer Overlay Networking}

Several VICOM applications are inherently many to many and require different performance constrains. Due to the limited processing capacities of the ad hoc network devices, it appears not much practical to implement a lot of routing enhancement, which optimally solve the specific applications requirements. Starting from this consideration, it is foreseeable that the network layer of devices will offer basic unicast routing functionalities (and in the best case, some coarse QoS mechanisms), while on-demand implementing the routing enhancements only for the application that it is currently running. Those enhancements are tightly connected with the application need, therefore their natural place on the protocol architecture is between the transport and the application layer. We refer this intermediate layer as overlay.

With this regard, VICOM research is focused on mechanisms for setup suitable peer to peer overlay networks facing with the many to many communication involved in collaborative work applications. Those mechanisms will take into account of the contextual information regarding the network and the application itself. Currently, overlay network solutions for MPEG 4 streaming over ad hoc network are under investigation.

\section{References}

[1] www.vicom-project.it

[2] A. Detti, F. Davide, G. Cortese, "eCASA: an Easy Context Aware System Architecture", to appear in Proc. IEEE Vehicular Technology Conference 2003

[3] R. Bruno, "802.11", VICOM Project, Technical report of first semester of WP4 activity, section 1.1, www.vicom-project.it

[4] G. Anastasi, E. Borgia, M. Conti, E. Gregori, "IEEE 802.11 Ad Hoc Network: Perfornamce Measurements", IIT Internal Report, January 2003 
[5] L. Badia, M. Zorzi, "On the Construction of Broadcast and Multicast Trees in Wireless Networks - Global vs. Local Energy Efficiency," in Proc. IEEE GLOBECOM2002, Taiwan, November 2002

[6] F. Cuomo, "Bluetooth", VICOM Project, Technical report of first semester of WP4 activity, section 2.1, www.vicom-project.it

[7] S. Basagni, R. Bruno, and C. Petrioli, "A performance comparison of scatternet formation protocols for networks of Bluetooth devices," in Proc. IEEE International Conference on Pervasive Computing and Communications, PerCom 2003, Forth Worth, TX, March 23 26 2003, pp. 341-350.

[8] A. Detti, P. Loreti, F. Mazenga, F. Vatalaro, "Performance Analysis of Bluetooth Inquiry and Page Procedures", in Proc. European Conference on Wireless Technology 2002 (ECWT2002), 26-27 September - Milan

[9] M. Zorzi, "Sensor Networks" VICOM Project, Technical report of first semester of WP4 activity, section 1.2, www.vicom-project.it

[10] G. Morabito, "End-to-End Protocols" VICOM Project, Technical report of first semester of WP4 activity, section 1.3, www.vicom-project.it 